\title{
Numerical Analysis of an Industrial Power Saving Mechanism in LTE
}

\author{
Scott Fowler, Georg Baravdish and Di Yuan
}

\section{Linköping University Post Print}

\section{Tweet}

N.B.: When citing this work, cite the original article.

Original Publication:

Scott Fowler, Georg Baravdish and Di Yuan, Numerical Analysis of an Industrial Power Saving Mechanism in LTE, 2014, IEEE International Conference on Communications (ICC), 2014, 1748-1753.

http://dx.doi.org/10.1109/ICC.2014.6883575

(C2013 IEEE. Personal use of this material is permitted. However, permission to reprint/republish this material for advertising or promotional purposes or for creating new collective works for resale or redistribution to servers or lists, or to reuse any copyrighted component of this work in other works must be obtained from the IEEE.

\section{http://ieeexplore.ieee.org/}

Postprint available at: Linköping University Electronic Press

http://urn.kb.se/resolve?urn=urn:nbn:se:liu:diva-111384 


\title{
Numerical Analysis of an Industrial Power Saving Mechanism in LTE
}

\author{
Scott Fowler*, George Baravdish ${ }^{\dagger}$ and Di Yuan* \\ * Mobile Telecommunications, Department of Science and Technology, Linköping University, Norrköping, Sweden \\ ${ }^{\dagger}$ Mathematics, Department of Science and Technology, Linköping University, Norrköping, Sweden
}

\begin{abstract}
The 4G standard Long Term Evolution (LTE) utilizes discontinuous reception (DRX) to extend the user equipments battery lifetime. DRX permits an idle UE to power off the radio receiver for two predefined sleep period and then wake up to receive the next paging message. Two major basic power saving models proposed to data are the 3GPP ETSI model and industrial DRX model proposed by Nokia. While previous studies have investigated power saving with the 3GPP ETSI models, the industrial DRX model has not been considered for analytical studies to date. Thus,there is a need to optimize the DRX parameters in the industrial model so as to maximize power saving without incurring network reentry and packet delays. In this paper, we take an overview of various static DRX cycles of the LTE/LTE-Advanced power saving mechanisms by modelling the system with bursty packet data traffic using a semi-Markov process. Using this analytical model, we will show the tradeoff relationship between the power saving and wake-up delay performance in the industrial model.
\end{abstract}

\section{INTRODUCTION}

The latest wireless communication technology being deployed in recent times is the $4 \mathrm{G}$ mobile technology known as Long Term Evolution (LTE) which had its early specifications in the 3GPP Release 8. The LTE Release 8 uses orthogonal frequency-division multiplexing (OFDM) for downlink multiple access and single-carrier frequency-division multiple access (SC-FDMA) for the uplink multiple access, both with a cyclic prefix (CP) which allows the users to benefit from a scalable $20 \mathrm{MHz}$ bandwidth [1]. Moreover enhancements like carrier aggregation were made and added in the LTE Release 10 or otherwise known as LTE-Advanced (LTE-A) which improved the bandwidth to $100 \mathrm{MHz}$. Other advancements like advanced antenna techniques (MIMO and SDMA), higher order modulation (64-QAM) and advanced coding systems have given end users' devices or user equipments (UE) the privilege of having peak data rates of $300 \mathrm{Mbit} / \mathrm{s}$ in LTE and $1 \mathrm{~Gb} / \mathrm{s}$ in LTE-A for downlink and 75Mbit/s in LTE and $500 \mathrm{Mbits} / \mathrm{s}$ in LTE-A for uplink communication. These advancements in mobile technology have made it necessary for receivers to have complex circuitry for computational purposes that drains the UE's battery power quickly [3].

High data rates and high bandwidth makes it possible for new services like voice, video and multimedia services (e.g. VoIP, video streaming, etc.) to be injected into the network. On the other hand, having these new services means the UE would require more power to be able to use all these new services. For keeping UEs powered up for longer period of time is an important but a difficult issue. One solution can be to increase the capacity of the UE's battery but that solution has its limitations. Another way of improving the battery life is to efficiently manage the power usage of the UE. Efficient power management can be done by turning off the UE during inactive periods and turning on again when there is a need for communication. This mechanism for power saving is known as discontinuous reception (DRX), which was first mentioned in 3GPP Release 7 but proper implementation specifications were provided in 3GPP Release 8 .

There are two major basic power saving models; the 3GPP ETSI model [2] and industrial DRX model proposed by Nokia [6], [11], [15]. While several previous works [4], [7]-[9], [13] has investigated power saving using the 3GPP ETSI models, the industrial DRX model has not been considered for analytical studies to date. Therefore, in this paper we conducted a study using the industrial model. We took an overview of the fixed DRX cycles with a semi-Markov process in order to evaluate the power saving and wake-up delay performance of LTE DRX mechanisms. The objective of the work is to help selecting the best parameters when LTE DRX is implemented with an industrial model.

\section{LTE AND THE DRX CONCEPT}

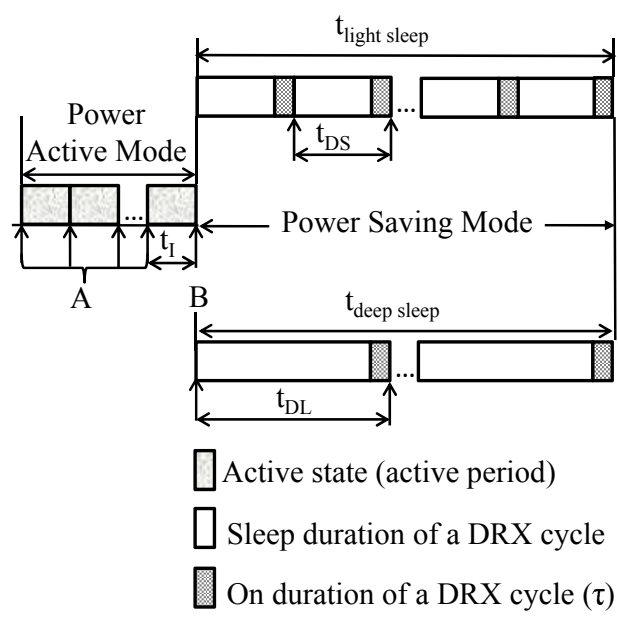

A. DRX Inactivity Timer activated $\left(\mathrm{t}_{\mathrm{I}}\right)$

B. DRX Inactivity Timer expired

Fig. 1: LTE DRX timing for UE receiver operations.

In 3GPP LTE Release 8 documentation a power saving mechanism called discontinuous reception (DRX) was specified to be implemented in LTE networks. To fully grasp the 


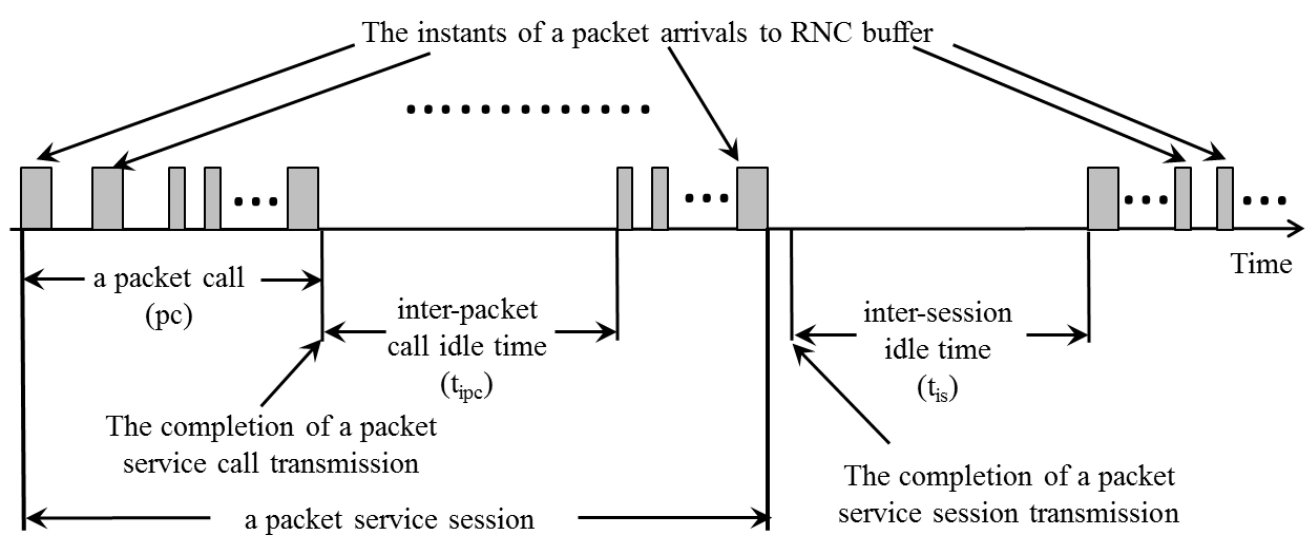

Fig. 2: ETSI traffic model [18]

notion of DRX it is essential understand the following terms that are related to DRX [2],

- Active state/On state: When an UE is actively monitoring the RF spectrum for data frames.

- Active Time: The time data packets are received by an UE.

- DRX Inactivity Timer: A timer used to initiate the sleep states.

- Sleep state/Off state: When an UE has powered down and not monitoring the RF spectrum.

- Listen state: Very short period of time when the UE powers up during DRX short cycle to monitor the RF spectrum for incoming PDCCH transmissions.

- DRX Short Cycle: A period of time when the UE is in sleep state but periodically transitions to the listen state to monitor the PDCCH for incoming data frames.

- DRX Short Cycle Timer: During the DRX short cycle the DRX Short Cycle Timer sets off the listen state.

- DRX Long Cycle: A time span longer than the DRX Short Cycle where an UE stays in sleep state and wakes up only at the end of the cycle to monitor the PDCCH.

- DRX Long Cycle Timer: During the DRX long cycle the DRX long Cycle Timer set off the listen state.

\section{An Analytical Model for LTE Power SaVing}

\section{A. Bursty Packet Traffic Model}

Traditional traffic models based on Poisson distribution have failed to predict data traffic as the results are often different from the actual observations [14]. To overcome this, the European Telecommunications Standards Institute (ETSI) proposed the ETSI packet traffic model. The characteristics of traditional and ETSI models are as follows:

- Traffic pattern:

- Traditional: traditional models consider data traffic pattern as Poisson distribution.

\begin{tabular}{llc}
\hline Parameter & Distribution & Mean Value \\
\hline Inter-session idle time, $t_{i s}$ & Exponential & $1 / \lambda_{i s}$ \\
Number of packet calls per session, $N_{p c}$ & Geometric & $\mu_{p c}$ \\
Inter-packet call idle time, $t_{i p c}$ & Exponential & $1 / \lambda_{i p c}$ \\
Number of packet calls per packet call, $N_{p}$ & Geometric & $\mu_{p}$ \\
Inter-packet arrival time, $t_{i p}$ & Exponential & $1 / \lambda_{i p}$ \\
\hline
\end{tabular}

TABLE I: Bursty data traffic model parameters

- ETSI: network data traffic is self-similar. Thus, heavy-tailed distributions, for example Pareto and Weibull distribution, are more appropriate. ETSI model follows the Pareto distribution.

- Focus of time scale:

- Traditional: typically, traditional models only focus on a very limited range of time scales and are shortrange dependent.

- ETSI: it focuses on extremely wide range of time scale. It considers the fact that real data traffic is bursty. Thus, ETSI model is long-range dependent.

The ETSI Packet Traffic Model has been adopted by previous studies to represent the network traffic patterns when analyzing the DRX mechanism [18] [4] [9]. Since this study will show the performance analysis of the enhancements made to the conventional 3GPP LTE DRX method so the same traffic model has been applied.

The Figure 2 depicts the ETSI packet traffic model, where it has been assumed that packet data traffic consists of several packet service sessions. Depending on different applications, each session contains one or more packets call [18]. Several packets might be generated during a packet call which comprises of bursty sequence of packets [5]. The streaming video may consists of one packet call per packet service session while the web browsing comprises sequences of packet call per packet service session. When the user equipment (UE) initiates a request for information, (for example downloading of a WWW page) a burst of packets will be transmitted to the UE through eNodeB after accepting its request. In this process, a current packet call will be completed while the 
eNodeB receives positive acknowledgement for the last packet of the packet call from the UE. After completion of a packet call the eNodeB starts the next new packet call. The time interval between the end of one packet call and the beginning of the next packet call is referred to as inter-packet call idle time, $\left(t_{i p c}\right)$. As a packet service session consist of one or more packet calls, after receiving all packets of these packet calls of the ongoing session, the UE will experience an idle time before beginning of next new session. The time between the end of last session and the beginning of new session is referred to as inter-session idle time, $t_{i s}$ [18].

The statistical distributions of the parameters of the LTE DRX model follow the recommendation of [5] [4] [18] and are summarized in Table [ Given the burstiness of the packet traffic, a typical ETSI packet traffic model is shown in Figure 2

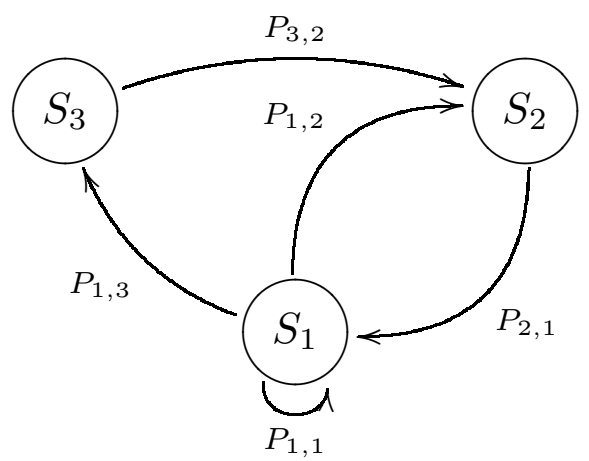

Fig. 3: Three-state Nokia ${ }^{T M}$ Power Consumption Model

The LTE DRX mechanism is a semi-Markov process [12] and is illustrated in Figure 3 The state transition diagram consists of three states, which are relevant to the three periods show in Figure 3 .

- State $S_{1}$ is a sequence of adjacent active time intervals corresponding to the entire duration of a single packet call transmission, i.e. the UE is in power active mode.

- State $S_{2}$ is a sequence of Light Sleep period $\left(t_{D S}\right)$ that are entered from the $S_{1}$, i.e. the UE follows DRX Short Cycles.

- State $S_{3}$ is a sequence of Deep Sleep period $\left(t_{D L}\right)$ which is entered from $S_{1}$ !

A new packet call can be viewed as continuation of the current session (Condition 1) or as the onset of a new session (Condition 2) depending on the time interval-arrive between two consecutive packet calls. The packet calls may be the inter-packet call idle time $\left(t_{i p c}\right)$ with probability $P_{p c}=1$ $1 / \mu_{p c}$ or the inter-session idle time $\left(t_{i s}\right)$ with probability $P_{s}$ $=1 / \mu_{p c}$. The probabilities take into account the memoryless property of a geometric distributions.

In relation with the ETSI packet traffic model, depending on the time interval between two consecutive packet calls, a new packet call may start during the ongoing session or

${ }^{1} 3$ GPP ETSI would have entered from $S_{2}$ after a defined number of DRX Short cycles $\left(t_{D S}\right)$ which is specify by the DRX Short Cycle Timer $\left(t_{N}\right)$. a new session will start where the packet call will be the first packet call of that session. A new packet call can be viewed as continuation of the current session (Condition 1) or as the onset of a new session (Condition 2) depending on the time interval-arrive between two consecutive packet calls. The packet calls may be the inter-packet call idle time $\left(t_{i p c}\right)$ with probability $P_{p c}=1-1 / \mu_{p c}$ or the inter-session idle time $\left(t_{i s}\right)$ with probability $P_{s}=1 / \mu_{p c}$. The probabilities take into account the memoryless property of a geometric distributions.

After defining the states of the new proposed DRX techniques, the transitions between the states are also defined through the semi-Markov process to obtain a embedded Markov chain and with the help of the Markov chain we can derive the state-transition probabilities $P_{i, j}$, where $i, j \in\{1,2$, $3\}$. Next, we derive these state transition probabilities. Next, we derive these state transition probabilities.

\section{B. State 1 to State 1, State 1 to State 2 and State 1 to State 3}

State $S_{1}$ contains $N_{p}$ inactivity period 2 . During the last inactivity period, if the PDCCH indicates the next packet call delivery happened before the DRX Inactivity Timer expires, the DRX Inactivity Timer is cancelled, another inactivity period is started and state $S_{1}$ is re-entered ( $t_{I}$ has not expired); otherwise, state $S_{2}$ or $S_{3}$ is entered when the DRX Inactivity Timer expires.

The probability that a new packet call begins before the expiration of $t_{I}$ is $q_{1}=\operatorname{Pr}\left[t_{i p c}<t_{I}\right]=1-\mathrm{e}^{-\lambda_{i p c} t_{I}}$ in Condition 1 and $q_{2}=\operatorname{Pr}\left[t_{i s}<t_{I}\right]=1-\mathrm{e}^{-\lambda_{i s} t_{I}}$ in Condition 2.

In the case of entering $S_{2}$ with a probability of $\delta_{2}=1 / \mu_{D R X}$ or a probability of $\delta_{3}=1-1 / \mu_{D R X}$ for the UE enter $S_{3}$. The probabilities are derived based on the memoryless property of a geometric distributions. Then we have:

$$
\begin{gathered}
P_{1,1}=\left(P_{p c} q_{1}+P_{s} q_{2}\right) \\
P_{1,2}=\left(P_{p c}\left(1-q_{1}\right)+P_{s}\left(1-q_{2}\right)\right) \delta_{2}
\end{gathered}
$$

and

$$
P_{1,3}=\left(P_{p c}\left(1-q_{1}\right)+P_{s}\left(1-q_{2}\right)\right) \delta_{3}
$$

$P_{3,2}$ : There is only one transition out of state $S_{3}$ to the state $S_{2}$, thus, we have $P_{3,2}=1$.

$P_{2,1}$ : There is only one transition out of state $S_{2}$ to the state $S_{1}$, thus, we have $P_{2,1}=1$.

\section{Transition Probability Matrix}

Next we expressed the probability matrix for the DRX models. We can express the embedded Markov chain transition probability matrix $\mathbf{P}=\left(P_{i, j}\right)$ as the following (4):

$$
\mathbf{P}=\left[\begin{array}{ccc}
p_{1,1} & p_{1,2} & p_{1,3} \\
1 & 0 & 0 \\
0 & 1 & 0
\end{array}\right]
$$

\footnotetext{
${ }^{2} N_{p}$ : Number of packets per packet call.
} 
Let $\pi_{i}(i \in\{1,2,3\})$ denote the probability of staying at state $S_{i}(i \in\{1,2,3\})$ of the embedded Markov chain. By using $\sum_{j=1}^{3} \pi_{i}=1$ and the balance equation $\pi_{i}=$ $\sum_{j=1}^{3} \pi_{j} P_{j, i}$, this gives us (5)

$$
\Pi=\left\{\left[\begin{array}{l}
\pi_{1}=\frac{1}{1+p_{1,2}+2\left(p_{1,3}\right)} \\
\pi_{2}=\frac{p_{1,2}+p_{1,3}}{1+p_{1,2}+2\left(p_{1,3}\right)} \\
\pi_{3}=\frac{p_{1,3}}{1+p_{1,2}+2\left(p_{1,3}\right)}
\end{array}\right]\right.
$$

The analysis of power saving (one of the performance evaluating parameter) involves the calculation of the time spent in the sleep modes, thus we proceed first in finding the time spent in all the available states. It is assumed that the holding time of the semi-Markov process at state $S_{i}$ to be $H_{i}(i \in\{1,2,3\})$. Now we proceed to derive $E\left[H_{i}\right]$ :

$\mathrm{E}\left[H_{1}\right]$ : In state $S_{1}$, the UE experiences a busy period $t_{B}{ }^{3}$ and then an interpacket call inactivity period $t_{I}$.

$$
E\left[H_{1}\right]=E\left[t_{B}\right]+E\left[t_{I}\right]
$$

From Wald's theorem [10]

$$
E\left[t_{B}\right]=E\left[N_{p}\right] E\left[\frac{1}{\lambda_{i p}}\right]=\frac{\mu_{p}}{\lambda_{x}}
$$

where $\mu_{p}$ is the number of packets calls within a packet service session and $\lambda_{x}$ is the Inter-packet arrive time.

If a packet arrives before the Inactivity Timer expires $\left(t_{i p c}<\right.$ $\left.t_{I}\right)$, then the Inactivity period equals the inter-packet call idle time, $t_{I}=t_{i p c}$; Otherwise the next packet arrives after the DRX Inactivity Timer has expired $\left(t_{I} \geq t_{i p c}\right)$. Therefore, we have $t_{I}=\min \left(t_{i p c}, t_{I}\right)$. Similarly, in Inter-session idle time $\left(t_{i s}\right)$, we have $t_{I}=\min \left(t_{i s}, t_{I}\right)$.

Therefore, we have for $t_{I}$ for $t_{i p c}$ and $t_{i s}$ yields:

$$
E\left[t_{I}\right]=P_{p c} E\left[\min \left(t_{i p c}, t_{I}\right)\right]+P_{s} E\left[\min \left(t_{i s}, t_{I}\right)\right]
$$

We obtain that:

$$
\begin{aligned}
E\left[\min \left(t_{i p c}, t_{I}\right)\right] & =\int_{x=0}^{\infty} \operatorname{Pr}\left[\min \left(t_{i p c}, t_{I}\right)>x\right] d x \\
& =\int_{x=0}^{t_{I}} \operatorname{Pr}\left[t_{i p c}>x\right] d x \\
& =\int_{x=0}^{t_{I}} e^{-\lambda_{i p c} x} d x=\left(\frac{1}{\lambda_{i p c}}\right)\left[1-e^{-\lambda_{i p c} t_{I}}\right]
\end{aligned}
$$

where $f\left(t_{i p c}\right)=\lambda_{i p c} e^{-\lambda_{i p c} t_{i p c}}$ is the PDF of the inter-packet call idle time $\mathrm{t}_{i p c}$. Likewise:

$$
E\left[\min \left(t_{i s}, t_{I}\right)\right]=\left(\frac{1}{\lambda_{i s}}\right)\left[1-e^{-\lambda_{i s} t_{I}}\right]
$$

Substitute equation (9) and (10) into (8)

$$
E\left[t_{I}\right]=\left(\frac{P_{p c}}{\lambda_{i p c}}\right)\left[1-e^{-\lambda_{i p c} t_{I}}\right]+\left(\frac{P_{s}}{\lambda_{i s}}\right)\left[1-e^{-\lambda_{i s} t_{I}}\right]
$$

\footnotetext{
${ }^{3} t_{B}$ : consists of the number of packet within a per packet call $\left(N_{p}\right)$.
}

Substitute equation (7) and (11) into (6)

$$
\begin{aligned}
E\left[H_{1}\right] & =\left(\frac{\mu_{p}}{\lambda_{x}}\right)+\left(\frac{P_{p c}}{\lambda_{i p c}}\right)\left[1-e^{-\lambda_{i p c} t_{I}}\right] \\
& +\left(\frac{P_{s}}{\lambda_{i s}}\right)\left[1-e^{-\lambda_{i s} t_{I}}\right]
\end{aligned}
$$

\section{Sleep States $\mathrm{H}_{2}$ AND $\mathrm{H}_{3}$}

State $S_{2}$ comprises a Light Sleep period consisting of $N_{D S}$ DRX Short Cycles. Therefore $E\left[H_{2}\right]=E\left[N_{D S}\right] t_{D S}$ :

$$
E\left[H_{2}\right]=\left(\frac{P_{p c}}{1-e^{-\lambda_{i p c} t_{D S}}}+\frac{P_{S}}{1-e^{-\lambda_{i s} t_{D S}}}\right) t_{D S}
$$

State $S_{3}$ comprises a Deep Sleep period consisting of $N_{D L}$ DRX Long Cycles. Also, $S_{3}$ will transition to $S_{2}$ as shown in Figure 3, this will require an extra DRX cycle. Therefore $E\left[H_{3}\right]=E\left[N_{D L}\right] t_{D L}+E\left[H_{2}\right]:$

$$
E\left[H_{3}\right]=\left(\frac{P_{p c}}{1-e^{-\lambda_{i p c} t_{D L}}}+\frac{P_{s}}{1-e^{-\lambda_{i s} t_{D L}}}\right) t_{D L}+E\left[H_{2}\right]
$$

\section{POWER SAVING Factor (PS)}

The power saving factor (PS) is equal to the probability that the semi-Markov process is at $S_{2}$ and $S_{3}$ (the percentage of time the UE has been kept in sleep mode or power saving mode). We note that, at the end of every DRX cycle, the UE must wake up for a short period $\tau$ so that it can listen to the paging information from the network. Therefore, the effective sleep duration is $t_{D S}^{\prime}=t_{D S}-\tau$ for the DRX Short Cycle and $t_{D L}^{\prime}=t_{D L}-\tau$ for the DRX Long Cycle. Also, when $S_{3}$ is transition to $S_{2}$, there is no listening to the paging information from the network for $S_{2}$. Therefore, we derived the following effective sleep time:

$$
\begin{gathered}
E\left[H_{2}^{\prime}\right]=\left(\frac{P_{p c}}{1-e^{-\lambda_{i p c} t_{D S}}}+\frac{P_{s}}{1-e^{-\lambda_{i s} t_{D S}}}\right) t_{D S}^{\prime} \\
E\left[H_{3}^{\prime}\right]=\left(\frac{P_{p c}}{1-e^{-\lambda_{i p c} t_{D L}}}+\frac{P_{s}}{1-e^{-\lambda_{i s} t_{D L}}}\right) t_{D L}^{\prime}+E\left[H_{2}\right]
\end{gathered}
$$

By substituting Equations (5), (12), (13), (14), (15) and (16) we derived:

$$
P S= \begin{cases}\frac{\pi_{2} E\left[H_{2}^{\prime}\right]}{\sum_{i=1}^{2} \pi_{i} E\left[H_{i}\right]} & \delta_{2}>0 \\ \frac{\pi_{3} E\left[H_{3}^{\prime}\right]}{\pi_{1} E\left[H_{1}\right]+\pi_{3} E\left[H_{3}\right]} & \delta_{3}>0\end{cases}
$$

A packet call transmission may begin whether we are in Deep Sleep or Light Sleep. The probability that a packet call delivery starts during the $i^{\text {th }}$ DRX Cycle is in a fixed DRX Cycles:

$$
p_{i}=\left\{\begin{array}{l}
P_{p c} e^{-\lambda_{i p c}(i-1) t_{D S}}\left(1-e^{-\lambda_{i p c} t_{D S}}\right) \\
+P_{s} e^{-\lambda_{i s}(i-1) t_{D S}}\left(1-e^{-\lambda_{i s} t_{D S}}\right), \quad \delta_{2}>0
\end{array}\right.
$$




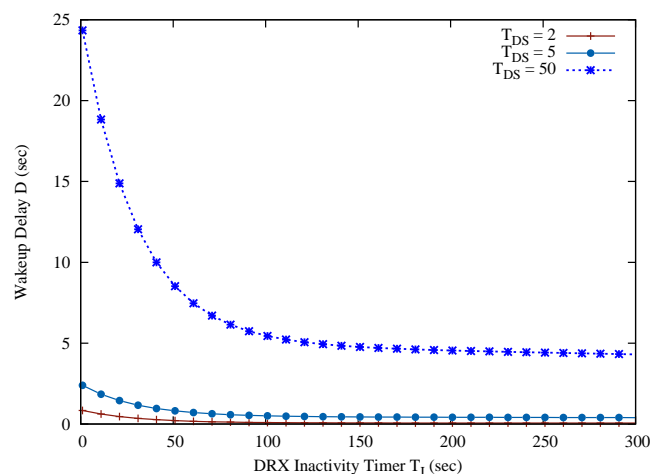

Fig. 4: LTE DRX Short Cycles on $T_{I}$ for Delay.

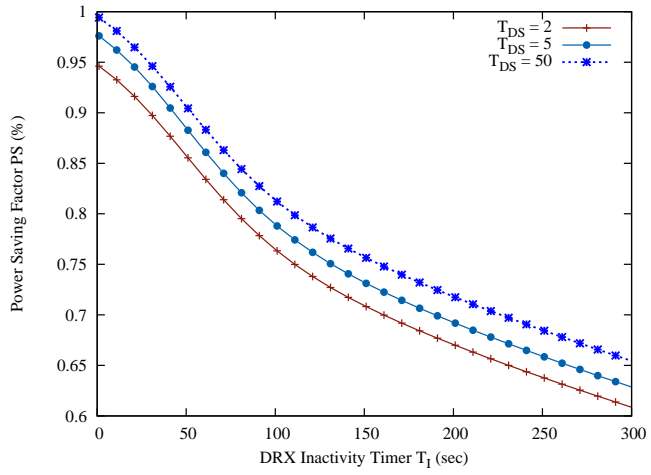

Fig. 5: LTE DRX Short Cycles on $T_{I}$ for Power.

$$
q_{i}=\left\{\begin{array}{l}
P_{p c} e^{-\lambda_{i p c}(i-1) t_{D L}}\left(1-e^{-\lambda_{i p c} t_{D L}}\right) \\
+P_{s} e^{-\lambda_{i s}(i-1) t_{D L}}\left(1-e^{-\lambda_{i s} t_{D L}}\right), \quad \delta_{3}>0
\end{array}\right.
$$

Next we derive the delay. The arrival event are random observer sleep durations due the packet call arrivals follow a Poisson distribution [16], [17], [19]. Substituting Equation (18) and (19) into Equation (20), this gives us the $E[D]$ :

$$
E[D]= \begin{cases}\sum_{i=1}^{\infty} p_{i} \frac{t_{D S}}{2} & \delta_{2}>0 \\ p_{i} \frac{t_{D S}}{2}+\sum_{i=1}^{\infty} q_{i} \frac{t_{D L}}{2} & \delta_{3}>0\end{cases}
$$

\section{NUMERICAL RESULtS}

The values of the parameters of the bursty packet data traffic model for the analytical model are as follows: $\lambda_{i p}=10$, $\lambda_{i p c}=1 / 30, \lambda_{i s}=1 / 2000, \mu_{p c}=5$, and $\mu_{p}=25$. Note, we keep the $t_{D S}$ and the $t_{D L}$ cycle the same sleep length in order to observe the behaviour in relation to power saving and delay. The first parameter with which we are evaluating is the DRX Inactivity Timer $\left(t_{I}\right)$ in Figures $4-7$. When the $t_{I}$ becomes larger, in the case of the $t_{D S} \mathrm{DRX}$ cycle, it is more likely that a packet call delivery occurs before the DRX Inactivity Timer expires resulting in fewer transition to the power saving mode. Since the number of transitions to the power saving mode are more infrequent, the impact of the amount of delayed packet call deliveries will be minor, consequence both power saving and delay are smaller. This is similiar in the case of the the $t_{D L}$ DRX cycle-

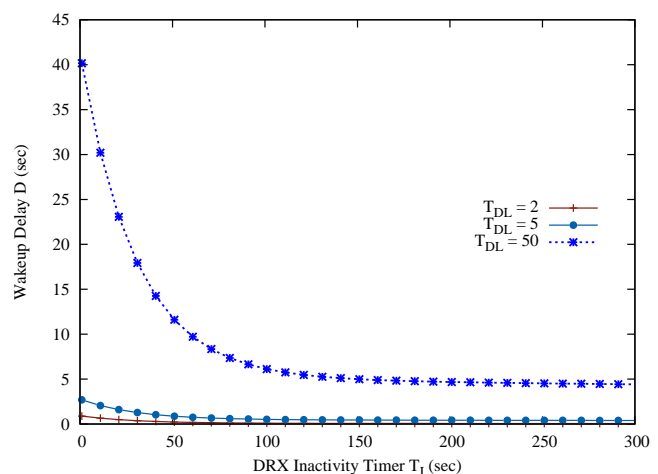

Fig. 6: LTE DRX Long Cycles on $T_{I}$ for Delay.

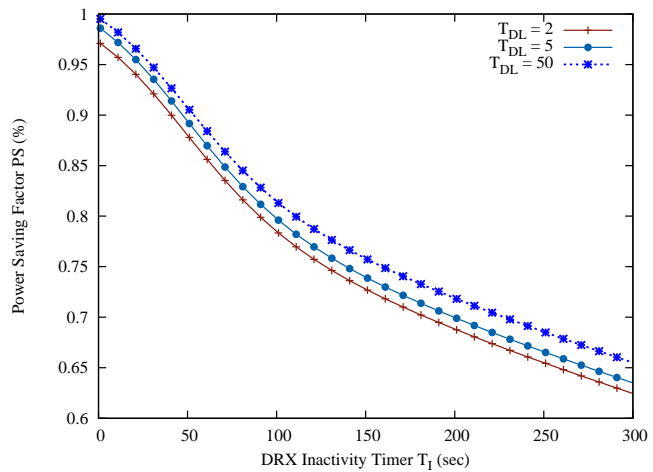

Fig. 7: LTE DRX Long Cycles on $T_{I}$ for Power.

The $t_{D L}$ DRX cycles have more power saving compared to the $t_{D S}$ DRX cycle. This is due to the extra $t_{D S}$ DRX cycle for the $t_{D L}$ DRX cycles, resulting in slightly longer power saving mode. Despite the increase in the power saving the values of the $t_{I}$ for the $t_{D L}$ DRX cycle follows the same behaviour as $t_{D S}$. The delay is decreased when $t_{I}$ is increased as a result of fewer transition to powering saving mode.

Next, we will look at the $t_{D S}$ DRX Short Cycle and the $t_{D L}$ DRX Long Cycle in relation to the power saving and delay in Figures 8 - 11 The power saving shown in Figures 9 and 11 are increasing for both $t_{D S}$ and $t_{D L}$, because the Sleep Cycles are longer and the "ON Duration is fixed". The $t_{D L}$ DRX Cycle has greater power saving since the extra DRX cycle also becomes longer. The longer DRX cycles means more effective sleep time per cycle, resulting in better power saving. As the Sleep Cycles are increasing size the wake-up delay in Figures 8 and 10 . The increased power saving factor inevitably affects the performance of the wake-up delay.

From the results, there is a performance trade-off relationship between power saving factor and wake-up delay as presented in Figures 8 - 11 The tradeoff is when there is an improvement in power saving, there is an opposite effect on the wake-up delay. The UE's decision on whether it will be Light DRX sleep cycles or Deep DRX sleep, but will have a direct affect wake-up delay performance. Therefore, when considering the DRX parameters, one should carefully according to the tradeoff between power saving factor and wake-up delay performance. 


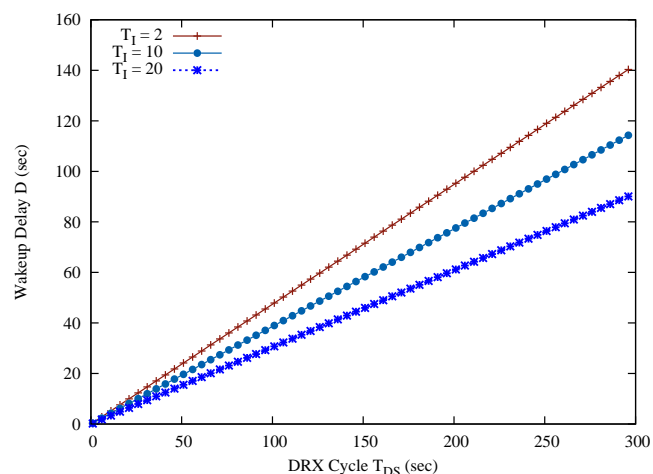

Fig. 8: LTE DRX Short Cycles on $T_{D S}$ for Delay.

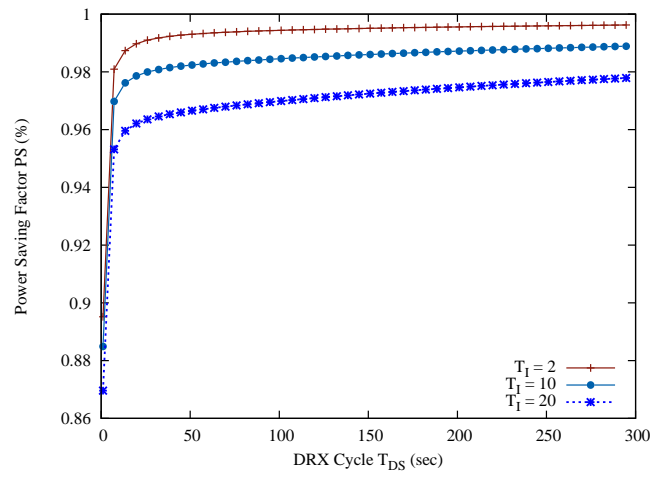

Fig. 9: LTE DRX Short Cycles on $T_{D S}$ for Power.

\section{CONCLUSION}

In this paper, we have generated a LTE industrial analytical models which were modeled with bursty packet data traffic using a semi-Markov process. Using an analytical modeling, the performance on power saving and wakeup delay was investigated and the trade-off relationship was illustrated when altering the three DRX parameters. Moreover, mobile phone manufacturer can also consider this type of model for $5 \mathrm{G}$ to achieve an efficient battery usage at a acceptable level of wakeup delay.

\section{ACKNOWLEDGMENT}

Scott Fowler was partially supported by the EC-FP7 Marie Curie CIG grant, Proposal number: 294182.

\section{REFERENCES}

[1] 3GPP TS 36.300. Evolved Universal Terrestrial Radio Access (E-UTRA) and Evolved Universal Terrestrial Radio Access (E-UTRAN); Overall description; Stage 2. TS 36.300, 3rd Generation Partnership Project (3GPP), September 2008 .

[2] 3GPP TS 36.321. Evolved Universal Terrestrial Radio Access (EUTRA); Medium Access Control (MAC) protocol specification. TS 36.321, 3rd Generation Partnership Project (3GPP), September 2008.

[3] C. Bontu and E. Illidge. Drx mechanism for power saving in lte. IEEE Communications Magazine, 47(6):48-55, June 2009.

[4] Lei Zhou et' al. Performance analysis of power saving mechanism with adjustable drx cycles in 3gpp lte. In IEEE Vehicular Technology Conference (VTC Fall), pages 1-5, September 2008.

[5] ETSI. Universal mobile telecommunications system (umts); selection procedures for the choice of radio transmission technologies of the umts. Technical Report UMTS 30.03, version 3.2.0, April 1998.

[6] S. Fowler. Study on power saving based on radio frame in lte wireless communication system using drx. In IEEE GLOBECOM Workshops (GC Wkshps), pages 1062 -1066, December 2011.

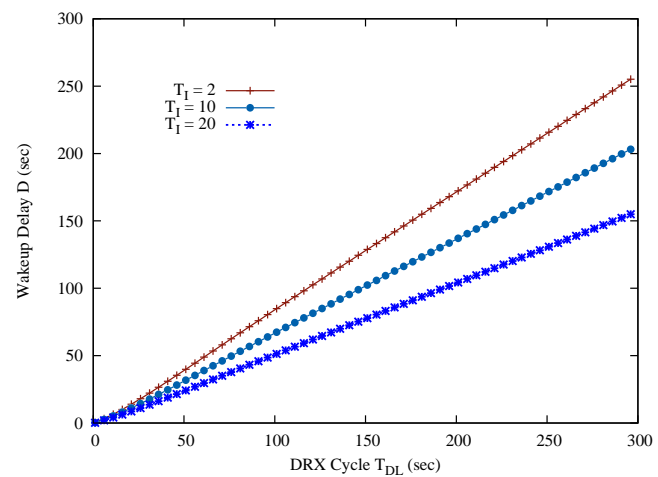

Fig. 10: LTE DRX Long Cycles on $T_{D L}$ for Delay.

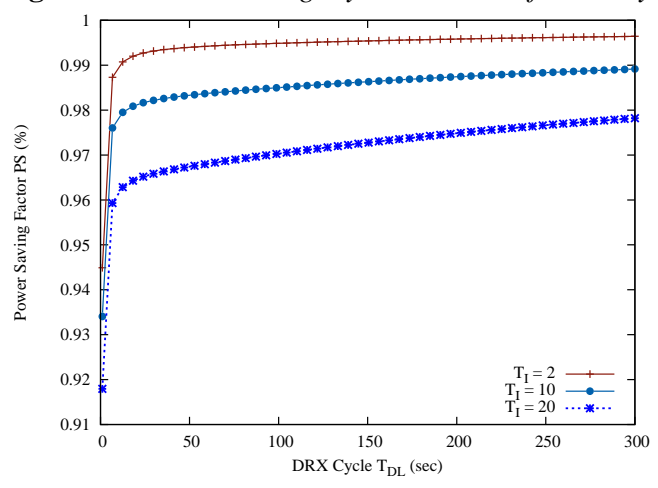

Fig. 11: LTE DRX Long Cycles on $T_{D L}$ for Power.

[7] S.C. Jha, A.T. Ko, and R. Vannithamby. Optimization of discontinuous reception (drx) for mobile internet applications over lte. In IEEE Vehicular Technology Conference (VTC-Fall), pages 1-5, 2012.

[8] Sunggeun Jin and D. Qiao. Numerical analysis of the power saving in 3gpp lte advanced wireless networks. Vehicular Technology, IEEE Transactions on, 61(4):1779-1785, 2012.

[9] Y.Y. Mihov, K.M. Kassev, and B.P. Tsankov. Analysis and performance evaluation of the drx mechanism for power saving in lte. In IEEE Convention of Electrical and Electronics Engineers in Israel (IEEEI), pages 520 - 524, November 2010

[10] Randolph Nelson. Probability, stochastic processes, and queueing theory: the mathematics of computer performance modeling. SpringerVerlag New York, Inc., New York, NY, USA, 1995.

[11] Nokia R2-071285. 3gpp ts 36.300. www.wireless-world-research.org, 3GPP TSG RAN WG2 LTE, March 2007.

[12] S. M. Ross. Stochastic Processes, 2nd Ed. John Wiley \& Sons, 1996.

[13] Scott Fowler and Ranjeet S. Bhamber and Abdelhamid Mellouk. Analysis of adjustable and fixed drx mechanism for power saving in lte/lteadvanced. IEEE International Conference on Communications (ICC 12), pages 10-15, June 2012.

[14] Walter Willinger, et al. Self-similarity through high-variability: Statistical analysis of ethernet lan traffic at the source level. IEEE/ACM Transactions on Networking, 5(1):71-86, 1997.

[15] J. Wigard, T. Kolding, Lars Dalsgaard, and C. Coletti. On the user performance of lte ue power savings schemes with discontinuous reception in lte. In Communications Workshops, 2009. ICC Workshops 2009 IEEE International Conference on, pages 1-5, June 2009.

[16] Y. Xiao. Energy saving mechanism in the ieee 802.16e wireless man. IEEE Communications Letters, 9(7):595 - 597, July 2005.

[17] Yang Xiao. Performance analysis of an energy saving mechanism in the ieee $802.16 \mathrm{e}$ wireless man. IEEE Consumer Communications and Networking Conference (CCNC), 1:406 - 410, January 2006.

[18] Shun-Ren Yang, Sheng-Ying Yan, and Hui-Nien Hung. Modeling umts power saving with bursty packet data traffic. IEEE Transactions on Mobile Computing, 6(12), December 2007.

[19] Yan Zhang, Yang Xiao, and V.C.M. Leung. Energy management analysis and enhancement in ieee 802.16e wirelessman. IEEE Transactions on Vehicular Technology, 58(7):3738-3752, September 2009. 